

В. В. Тен, Полиномиальные первые интегралы систем с гироскопическими силами, Матем. заметки, 2000, том 68, выпуск 1, 151-153

DOI: https://doi.org/10.4213/mzm932

Использование Общероссийского математического портала Math-Net.Ru подразумевает, что вы прочитали и согласны с пользовательским соглашением http://www . mathnet.ru/rus/agreement

Параметры загрузки:

IP : 54.162 .27 .143

26 апреля 2023 г., $14: 47: 38$






\title{
ПОЛИНОМИАЛЬНЫЕ ПЕРВЫЕ ИНТЕГРАЛЫ СИСТЕМ С ГИРОСКОПИЧЕСКИМИ СИЛАМИ
}

\author{
B. В. Тен
}

1. Введение. Основной результат. Топологические препятствия к существованию полиномиальных по импульам первых интегралов механических систем впервые были изучены в работе [1]. Эти исследования для случая геодезических потоков на двумерном торе продолжены в [2]: показано, что спектр Фурье (его определение приводится ниже) потенциала вполне интегрируемой натуральной системы на двумерном торе лежит на двух перпендикулярных прямых (это утверждение доказано в случае, когда потенциал является тригонометрическим полиномом, и высказана гипотеза для произвольного аналитического потенциала).

В настоящей работе установлен критерий интегрируемости для задачи о движении точки по плоскому двумерному тору под действием гироскопических сил, заданных аналитической 2-формой. Постановка задачи принадлежит В.В.Козлову. В работе [3] доказано (даже в более общей ситуации), что интеграл формы гироскопических сил по конфигурационному пространству равен нулю.

Пусть гироскопические силы заданы 2 -формой $\lambda\left(x_{1}, x_{2}\right) d x_{1} \wedge d x_{2}$, кинетическая энергия-квадратичной формой $\left(y_{1}^{2}+y_{2}^{2}\right) / 2$ (здесь $x_{i}$ - угловые координаты на торе, $y_{i}$ - канонически сопряженные им импульсы, а $\lambda\left(x_{1}, x_{2}\right)$ - двоякопериодическая аналитическая функция). Из доказательства теоремы будет видно, что в качествекинетической энергии можно взять любую квадратичную форму с постоянными коэффициентами $\left(a y_{1}^{2}+b y_{1} y_{2}+c y_{2}^{2}\right) / 2$, после чего подходящей линейной заменой координат на накрьвающей привести ее к сумме квадратов импульсов.

Уравнения движения имеют вид

$$
\begin{aligned}
& \dot{x}_{1}=y_{1}, \quad \dot{x}_{2}=y_{2}, \\
& \dot{y}_{1}=\lambda\left(x_{1}, x_{2}\right) y_{2}, \quad \dot{y}_{2}=-\lambda\left(x_{1}, x_{2}\right) y_{1} .
\end{aligned}
$$

Они, конечно, допускают интеграл энергии $y_{1}^{2}+y_{2}^{2}$.

ОПРЕДЕЛЕниЕ. Спектром Фурье функции $f\left(x_{1}, x_{2}\right)$, периодической по обоим аргументам, назьвается подмножество целочисленной решетки $\left\{(k, m) \in \mathbb{Z}^{2} \mid f_{k m} \neq 0\right\}$, где $f_{k m}$ - коэффициенты разложения этой функции в ряд Фурье.

В указанных предположениях справедлива

Теорема. Система (0) допускает дополнительный полиномиальный по импульсам интеграл тогда и только тогда, когда спектр Фурье функи,ии $\lambda\left(x_{1}, x_{2}\right)$ лежит на одной прямой, проходящей через начало координат, и среднее значение этой функиии по конфигурационному тору равно нулю (это эквивалентно существованию чиклической переменной).

СледствиЕ. Степень любого дополнительного неприводимого интеграла такой системы равна единиче.

2. Доказательство теоремы. Перейдем к новой "полярной" системе координат $\left(x_{1}, x_{2}, \varphi\right.$, $r)$ в $T^{*} \mathbb{T}^{2}$ :

$$
y_{1}=r \cos \varphi, \quad y_{2}=r \sin \varphi .
$$

Уравнения движения примут вид

$$
\begin{aligned}
\dot{x}_{1} & =r \cos \varphi, & \dot{x}_{2} & =r \sin \varphi, \\
\dot{\varphi} & =-\lambda\left(x_{1}, x_{2}\right), & \dot{r} & =0 .
\end{aligned}
$$

Работа выполнена при финансовой поддержке Российского фонда фундаментальных исследований, грант № 99-01-00953, и фонда INTAS, грант № 97-10771. 
Пусть $F\left(x_{1}, x_{2}, y_{1}, y_{2}\right)$ - полиномиальньй по импульсам первый интеграл. Будем считать, что он не делится на тривиальньй первый интеграл $y_{1}^{2}+y_{2}^{2}$. В новой системе координат его можно представить в следующем виде:

$$
\begin{gathered}
F\left(x_{1}, x_{2}, \varphi, r\right)=\sum_{k=0}^{n} \sum_{m \in M_{k}} f_{k m}\left(x_{1}, x_{2}\right) r^{k} e^{i \varphi m}, \\
M_{k}=\{m \in \mathbb{Z} \mid-k \leqslant m \leqslant k,(k+m) \bmod 2=0\} .
\end{gathered}
$$

Все коэффициенты с индексами, не попавшими в условия суммирования, равны нулю.

Приравнивая нулю производную $F$ в силу системы (1), получаем уравнения на коэффициенты

$$
\frac{\partial f_{k-1 m-1}}{\partial z}-i m \lambda f_{k m}+\frac{\partial f_{k-1 m+1}}{\partial \bar{z}}=0
$$

В дальнейшем для удобства записи предполагается, что интеграл имеет степень $n+1$.

Введем обозначение: $g_{k}=f_{k k}$. Тогда функции $g_{k}$ удовлетворяют цепочке соотношений:

$$
\begin{gathered}
\frac{\partial g_{n+1}}{\partial z}=0, \quad \frac{\partial g_{n}}{\partial z}=i(n+1) \lambda g_{n+1}, \quad \frac{\partial g_{n-1}}{\partial z}=i n \lambda g_{n} \\
\frac{\partial g_{n-2}}{\partial z}=i(n-1) \lambda g_{n-1}, \quad \ldots, \quad \frac{\partial g_{1}}{\partial z}=i 2 \lambda g_{2}, \quad \frac{\partial g_{0}}{\partial z}=i \lambda g_{1} .
\end{gathered}
$$

В силу ограниченности коэффициентов $g_{n+1}-$ произвольная постоянная. Простым поворотом системы координат $\left(x_{1}, x_{2}\right)$ и нормировкой интеграла можно сделать этот коэффициент равным единице (новые координаты, вообще говоря, не задают двояко-периодических координат на торе, но нас это не должно беспокоить, так как дальнейшие рассуждения применяются к системе, поднятой на накрывающую плоскость $\left.\mathbb{R}^{2}\left\{x_{1}, x_{2}\right\}\right)$. Таким образом, первое уравнение (2) можно заменить на $g_{n+1}=1$, а второе на $\partial g_{n} / \partial z=i(n+1) \lambda$, откуда сразу следует равенство нулю среднего функции $\lambda$.

Если $g_{n+1}=0$, то начнем с ненулевого коэффициента с максимальньм номером. Все $g_{k}$ не могут быть равными нулю, так как мы предположили, что интеграл не делится на $y_{1}^{2}+y_{2}^{2}$.

Перемножая второе и третье уравнения системы (2), имеем

откуда получаем

$$
\frac{\partial\left(g_{n}^{2}\right)}{\partial z}=\frac{2(n+1)}{n} \frac{\partial g_{n-1}}{\partial z}
$$

$$
g_{n-1}=\frac{n}{2(n+1)} g_{n}^{2}+C_{n-1}
$$

Вообще, для произвольного $k$

$$
\frac{\partial\left(g_{k}^{2}\right)}{\partial z}=\frac{2(k+1)}{k} \frac{\partial g_{k-1}}{\partial z} g_{k+1}
$$

По индукции можно доказать, что все функции $g_{k}$ выражаются через $g_{n}: g_{k}=G_{k}\left(g_{n}\right)$, где $G_{k}(w)$ - голоморфные всюду, за исключением счетного множества точек, функции.

Предположим, это справедливо для коэффициентов с номерами $\geqslant k$. Из формулы (3) получаем

$$
\frac{\partial g_{k-1}}{\partial z}=\frac{k}{2(k+1)} \frac{\partial\left(g_{k}^{2}\right)}{\partial z} \frac{1}{g_{k+1}}=\frac{k}{k+1} \frac{G_{k}\left(g_{n}\right)}{G_{k+1}\left(g_{n}\right)} \frac{\partial G_{k}\left(g_{n}\right)}{\partial g_{n}} \frac{\partial g_{n}}{\partial z}=\frac{\partial I\left(g_{n}\right)}{\partial g_{n}} \frac{\partial g_{n}}{\partial z},
$$

где $I(w)$ - первообразная функции

$$
\frac{k}{k+1} \frac{G_{k}(w)}{G_{k+1}(w)} \frac{\partial G_{k}(w)}{\partial w} .
$$

Следовательно, $g_{k-1}=I\left(g_{n}\right)+C_{k-1}=G_{k-1}\left(g_{n}\right)$.

Таким образом, $g_{0}(z)=G_{0}\left(g_{n}(z)\right)$, причем в силу вещественности разлагаемой функции $\operatorname{Im} G_{0}\left(g_{n}(z)\right) \equiv 0$. Функция $G_{0}(z)$ не постоянна, так как в противном случае либо $\lambda=0$, либо все остальные $g_{k}=0$, а в этом случае $F-g_{0}$ делится нацело на кинетическую энергию.

Для окончания доказательства нам потребуется 
Лемма. Пусть $f(z)$ и $\lambda(z)$ - вещественно-аналитические функиии двух переменных $x_{1}$ и $x_{2}\left(z=x_{1}+i x_{2}\right)$ такие, что $R(f(z))$ и $i \partial f(z) / \partial z \equiv-\lambda(z)$ - чисто вещественнье функиии, где $R(w)$ - непостоянная голоморфная всюду кроме дискретного множества функиия; кроме того, функиии $f$ и $\lambda$ не имеют особенностей на всей плоскости. Тогда существует линейная ортогональная замена координат $\left(x_{1}, x_{2}\right) \mapsto\left(X_{1}, X_{2}\right)$ такая, что $\lambda$ не зависит от переменной $X_{2}$.

Это утверждение доказано в п. 3.

Для завершения доказательства необходимости осталось, учитывая второе уравнение системы $(2)$ (при $g_{n+1}=1$ ), применить лемму и вернуться на накрьваемьй тор.

Докажем достаточность. Пусть $\lambda=\lambda\left(x_{2}\right)$ и $\int_{\mathbb{T}^{2}} \lambda d x_{1} d x_{2}=0$. Рассмотрим линейную по импульсам функцию $-\mu\left(x_{2}\right)+y_{1}$, где $\mu$ - первообразная $\lambda$ по $x_{2}$. Нетрудно проверить, что это первый интеграл, периодический по $x_{2}$.

Теорема доказана.

3. Доказательство леммы. Уравнение $\operatorname{Im}(R(u+i v))=0$ определяет наплоскости $\mathbb{R}^{2}\{u, v\}$ некоторую аналитическую кривую. Можно считать, что $v=S(u)$, где $S$ - некоторая вещественно-аналитическая функция одной переменной (по крайней мере локално). Тогда $\partial v / \partial x_{1}=$ $S^{\prime}(u) \partial u / \partial x_{1}, \partial v / \partial x_{2}=S^{\prime}(u) \partial u / \partial x_{2}$, а условия леммы

$$
\frac{\partial u}{\partial x_{1}}+\frac{\partial v}{\partial x_{2}}=0, \quad \frac{\partial v}{\partial x_{1}}-\frac{\partial u}{\partial x_{2}}=\lambda
$$

можно переписать в виде

$$
\frac{\partial u}{\partial x_{1}}+S^{\prime}(u) \frac{\partial u}{\partial x_{2}}=0, \quad S^{\prime}(u) \frac{\partial u}{\partial x_{1}}-\frac{\partial u}{\partial x_{2}}=\lambda .
$$

Рассмотрим первое уравнение системы (4). Пусть $u\left(x_{1}, x_{2}\right)$ - некоторое его нетривиальное частное решение, удовлетворяющее условиям леммы. Предположим $L_{\mathrm{c}}=\left\{\left(x_{1}, x_{2}\right) \mid u\left(x_{1}, x_{2}\right)=c\right\}-$ неособая кривая уровня $(c=$ const $)$; по лемме Сарда множество таких кривых имеет полную меру. Вектор $\left(1, S^{\prime}(u)\right)$ ортогонален вектору градиента и является постоянным на $L_{c}$, так как $S^{\prime}$ зависит только от значения $u$. Следовательно, линия уровня $L_{c}-$ прямая. Аналогичные рассуждения можно провести и для близких кривых $L_{c+\varepsilon}$. Ясно, что эти прямые не должны пересекаться, откуда следует, что они параллельны и множитель $S^{\prime}=$ const (в действительности можно показать, что эта постоянная - нуль).

Поворотом исходной системы координат в неболшшой окрестности $L_{c}$ можно ввести новые координаты $\left(X_{1}, X_{2}\right)$, в которых система (4) записьвается как

$$
\frac{\partial u}{\partial X_{1}}=\lambda, \quad \frac{\partial u}{\partial X_{2}}=0 .
$$

Следовательно, $\lambda$ не зависит от $X_{2}$.

В силу аналитичности функции $\lambda\left(x_{1}, x_{2}\right)$ такие координаты существуют на всей плоскости.

Лемма доказана.

Автор благодарит В. В. Козлова за внимание к работе.

\section{СПИСОК ЦИТИРОВАННОЙ ЛИТЕРАТУРЫ}

1. Козлов В.В., Трещев Д. В. // Матем. сб. 1988. Т. 135. № 1. С. 119-138. 2. Козлов В.В., Денисова Н. В. // Матем. сб. 1993. Т. 184. №9. С. 125-148. 3. Болотин С. В. // Вестн. МГУ. Сер. 1. Матем., мех. 1984. №6. С. 75-82. 